\title{
Orographic drag associated with lee waves trapped at an inversion
}

\section{Article}

Published Version

Teixeira, M. A. C., Argaín, J. L. and Miranda, P. M. A. (2013) Orographic drag associated with lee waves trapped at an inversion. Journal of the Atmospheric Sciences, 70 (9). 29302947. ISSN 1520-0469 doi: https://doi.org/10.1175/JAS-D-120350.1 Available at https://centaur.reading.ac.uk/32960/

It is advisable to refer to the publisher's version if you intend to cite from the work. See Guidance on citing.

Published version at: http://dx.doi.org/10.1175/JAS-D-12-0350.1

To link to this article DOI: http://dx.doi.org/10.1175/JAS-D-12-0350.1

Publisher: American Meteorological Society

All outputs in CentAUR are protected by Intellectual Property Rights law, including copyright law. Copyright and IPR is retained by the creators or other copyright holders. Terms and conditions for use of this material are defined in the End User Agreement.

\section{www.reading.ac.uk/centaur}

\section{CentAUR}

Central Archive at the University of Reading

Reading's research outputs online 


\title{
Orographic Drag Associated with Lee Waves Trapped at an Inversion
}

\author{
Miguel A. C. TeiXeirA \\ Department of Meteorology, University of Reading, Reading, United Kingdom \\ JosÉ LUIS ARGAÍN \\ Department of Physics, University of Algarve, Faro, Portugal \\ Pedro M. A. Miranda \\ IDL, University of Lisbon, Lisbon, Portugal
}

(Manuscript received 18 December 2012, in final form 8 April 2013)

\begin{abstract}
The drag produced by 2D orographic gravity waves trapped at a temperature inversion and waves propagating in the stably stratified layer existing above are explicitly calculated using linear theory, for a two-layer atmosphere with neutral static stability near the surface, mimicking a well-mixed boundary layer. For realistic values of the flow parameters, trapped-lee-wave drag, which is given by a closed analytical expression, is comparable to propagating-wave drag, especially in moderately to strongly nonhydrostatic conditions. In resonant flow, both drag components substantially exceed the single-layer hydrostatic drag estimate used in most parameterization schemes. Both drag components are optimally amplified for a relatively low-level inversion and Froude numbers $\mathrm{Fr} \approx 1$. While propagating-wave drag is maximized for approximately hydrostatic flow, trapped-lee-wave drag is maximized for $l_{2} a=O(1)$ (where $l_{2}$ is the Scorer parameter in the stable layer and $a$ is the mountain width). This roughly happens when the horizontal scale of trapped lee waves matches that of the mountain slope. The drag behavior as a function of Fr for $l_{2} H=0.5$ (where $H$ is the inversion height) and different values of $l_{2} a$ shows good agreement with numerical simulations. Regions of parameter space with high trapped-lee-wave drag correlate reasonably well with those where lee-wave rotors were found to occur in previous nonlinear numerical simulations including frictional effects. This suggests that trapped-lee-wave drag, besides giving a relevant contribution to low-level drag exerted on the atmosphere, may also be useful to diagnose lee-rotor formation.
\end{abstract}

\section{Introduction}

Although the troposphere generally has a positive static stability, supporting the propagation of internal gravity waves, it is often characterized by a neutrally stratified layer near the surface, capped by a temperature inversion (associated, for example, with a convective, or otherwise well-mixed, boundary layer). This leads to the possibility of occurrence of lee waves trapped at this inversion in addition to waves propagating vertically in the stably stratified layer existing above.

Corresponding author address: Miguel A. C. Teixeira, Department of Meteorology, University of Reading, Earley Gate, P.O. Box 243, Reading RG6 6BB, United Kingdom.

E-mail: m.a.teixeira@reading.ac.uk
Most of the available studies on topographic gravity waves in the atmosphere have focused on internal waves, which encompass both vertically propagating waves (Smith 1980; Broutman et al. 2002; Teixeira and Miranda 2006, 2009) and lee waves trapped in a layer near the surface due to a decrease of the Scorer parameter with height (Doyle and Durran 2002; Broutman et al. 2003; Stiperski and Grubišić 2011; Teixeira et al. 2013). Waves trapped at an inversion, however, are of a different nature, being surface waves essentially similar to those propagating at an interface separating fluids with significantly different densities (e.g., ocean waves at an air-water interface).

It is known that all of these types of waves (vertically propagating waves, lee waves trapped in a layer, or lee waves trapped at an interface) produce a drag force (Baines 1995). Since trapped lee waves, in particular, are intrinsically nonhydrostatic, having relatively small 
horizontal scales (e.g., Wurtele et al. 1996), their parameterization in all but the highest-resolution numerical models will remain an issue for some time. Being trapped near the surface, these waves likely give a significant contribution to the low-level drag exerted on the atmosphere (Teixeira et al. 2013). Additionally, since gravitywave drag is an integral property of the flow, it presumably provides valuable indications concerning the intensity of the waves and their likeliness to break, being affected by flow configurations such as downslope windstorms and lee rotors, all of which are important for aviation safety (e.g., Darby and Poulos 2006).

However, the theories for gravity waves in continuously stratified fluids and in fluids with various layers of different density have been developed separately, often with different applications in mind. For that reason, in Baines (1995) there is no apparent connection between the drag produced by one-layer, constant-density flow with a free surface and continuously stratified flow over topography (the parameters on which these forces depend are different). In the case of a stably stratified semiinfinite layer over a neutrally stratified layer capped by an inversion, treated by Vosper (2004), both types of drag should exist.

Nevertheless, Vosper (2004) only calculated using linear theory the resonant wavelength of lee waves trapped at the inversion and the flow structure far away from the topography, as done originally by Scorer (1949) for a stratified two-layer atmosphere. While Scorer (1949) briefly addressed in his calculations the case of an atmosphere with a neutrally stratified layer near the surface (see also Scorer 1953, 1954), this part of his work was to a large extent overlooked. Interest in this kind of flow configuration was only reawakened by the recent field measurements of rotors in the Falkland Islands (Mobbs et al. 2005; Doyle and Durran 2007), where the lee waves responsible for the rotors appeared to be trapped at an inversion topping a neutrally stratified layer (Sheridan and Vosper 2006b,a). Studying how such flow configurations affect gravity-wave drag is relevant for understanding boundary layer effects on that force, but has not been done until now. Rather, previous studies were mostly concerned with the effect of boundary layer turbulence on wave drag (Chimonas and Nappo 1989; Grisogono 1994).

Recently, Teixeira et al. (2013) used linear theory to study the behavior of the trapped-lee-wave drag and propagating-wave drag for a two-layer atmosphere akin to that of Scorer (1949) (i.e., with a Scorer parameter that is higher near the surface and lower aloft). In the present study, the same will be done for the static stability profile considered by Vosper (2004). While linear theory is known to severely underestimate the disturbances associated with trapped lee waves for sufficiently high mountains (Vosper 2004), it can provide a benchmark for the behavior of the drag, against which existing numerical simulations may be compared and new ones planned in a more rational way. However, this benchmark has not been obtained until now for the present flow configuration. Additionally, and as mentioned above, the linear drag estimate probably gives useful qualitative indications about the intensity of the flow associated with trapped lee waves and vertically propagating waves and their dynamical significance.

In this study, the drag associated with lee waves trapped at an inversion capping a neutrally stratified layer and with waves propagating in the stably stratified layer aloft will be investigated. As in Teixeira et al. (2013), it will be seen that the trapped-lee-wave drag may be comparable to the propagating-wave drag, and larger than the hydrostatic one-layer reference drag. However, in contrast with the findings of Teixeira et al. (2013) and as discussed by Vosper (2004), only one trapped-lee-wave mode exists. This means that only one drag maximum exists in parameter space.

In section 2, the theoretical model used to perform the calculations is described. In section 3, the drag behavior as a function of the input parameters is illustrated and compared with new numerical simulations, as well as with those of Vosper (2004). Finally, section 4 contains a summary of the main findings of the present study.

\section{A two-layer drag model}

Consider steady uniform flow over an isolated 2D mountain ridge with relatively small elevation. Assume also that the flow is of sufficiently large scale to be approximately inviscid, but of sufficiently small scale for the rotation of Earth to be negligible. The equations of motion with the Boussinesq approximation may be linearized with respect to the (steady) disturbances associated with the atmospheric waves generated by the ridge, and combined into a single equation for the vertical velocity perturbation $w$. If a Fourier transformation in the $x$ direction is applied to the resulting equation, it yields (Lin 2007)

$$
\frac{d^{2} \hat{w}}{d z^{2}}+\left(l^{2}-k^{2}\right) \hat{w}=0,
$$

where $\hat{w}$ is the Fourier transform of $w, k$ is the horizontal wavenumber of the waves, $z$ is height, and $l$ is the Scorer parameter, defined by

$$
l^{2}=\frac{N^{2}}{U^{2}}-\frac{1}{U} \frac{d^{2} U}{d z^{2}}
$$


where $N^{2}(z)$ is the static stability of the atmosphere and $U(z)$ is the mean velocity of the incoming flow, assumed to be perpendicular to the ridge (i.e., along the $x$ direction).

Following Vosper (2004), it is assumed that $U$ is constant, thereby neglecting boundary layer effects on the mean velocity profile, but a two-layer structure is assumed for the mean static stability, with the lower layer extending between the surface $z=0$ and $z=H$. The mean static stability is assumed to be zero in the lower layer, for $0<z<H$, and takes the positive value $N_{2}^{2}$ in the upper layer, for $z>H$, being infinite at $z=H$. Although the Richardson number is thus zero in the lower layer, the effect of turbulence is neglected. The inversion that is assumed to exist at $z=H$ is quantified by the corresponding potential temperature jump $\Delta \theta$, or alternatively by the reduced gravity $g^{\prime}=g \Delta \theta / \theta_{0}$, where $\theta_{0}$ is a reference potential temperature (assumed to be constant) and $g$ is the acceleration of gravity. form

The solution to (1) in the lower layer $z<H$ is of the

$$
\hat{w}_{1}=a_{1} e^{-|k| z}+b_{1} e^{|k| z},
$$

corresponding to evanescent waves. In the upper layer $z>H$, the waves may either be vertically propagating or evanescent, corresponding, respectively, to

$$
\begin{array}{ll}
\hat{w}_{2}=a_{2} e^{i m_{2} z} & \text { if } \quad k^{2}<l_{2}^{2}, \\
\hat{w}_{2}=c_{2} e^{-n_{2} z} & \text { if } \quad k^{2}>l_{2}^{2},
\end{array}
$$

where the coefficients $a_{1}, b_{1}, a_{2}$, and $c_{2}$ are functions of $k, m_{2}=\left(l_{2}^{2}-k^{2}\right)^{1 / 2} \operatorname{sgn}(U k)$, and $n_{2}=\left(k^{2}-l_{2}^{2}\right)^{1 / 2}$, where $l_{2}=N_{2} / U$.

In this model setup, the boundary conditions that the solutions (3)-(5) must satisfy result from the fact that the flow must be tangential to the topography at the surface (free-slip boundary condition) and that the velocity and pressure perturbations must be continuous at $z=H$. These boundary conditions are expressed as

$$
\begin{gathered}
\hat{w}_{1}(z=0)=i U k \hat{h}, \\
\hat{w}_{1}(z=H)=\hat{w}_{2}(z=H), \\
\frac{d \hat{w}_{1}}{d z}(z=H)-\frac{d \hat{w}_{2}}{d z}(z=H)=\frac{g^{\prime}}{U^{2}} \hat{w}_{1}(z=H),
\end{gathered}
$$

where $\hat{h}$ is the Fourier transform of the surface elevation. The last boundary condition was derived, for example, by Vosper (2004) and assumes that the density jump at the interface separating the two atmospheric layers is relatively small (i.e., $\Delta \theta / \theta_{0} \ll 1$ ). Additionally, the radiation boundary condition states that the wave energy must decay or propagate upward as $z \rightarrow+\infty$. This is already implicitly taken into account in (4)-(5), along with the definitions of $m_{2}$ and $n_{2}$ presented above.

If the boundary conditions (6)-(8) are imposed on the solutions (3)-(5), the coefficients $a_{1}, b_{1}, a_{2}$, and $c_{2}$ may be determined explicitly. For waves that propagate in the upper-atmospheric layer-that is, for $k^{2}<l_{2}^{2}-a_{1}$ and $b_{1}$ take the form

$$
\begin{aligned}
a_{1}= & \frac{1}{2} i U k \hat{h} \\
& \times \frac{e^{|k| H}\left(|k| H-i m_{2} H-g^{\prime} H / U^{2}\right)}{|k| H \cosh (|k| H)-\left(i m_{2} H+g^{\prime} H / U^{2}\right) \sinh (|k| H)},
\end{aligned}
$$

$$
\begin{aligned}
b_{1}= & \frac{1}{2} i U k \hat{h} \\
& \times \frac{e^{-|k| H}\left(|k| H+i m_{2} H+g^{\prime} H / U^{2}\right)}{|k| H \cosh (|k| H)-\left(i m_{2} H+g^{\prime} H / U^{2}\right) \sinh (|k| H)} .
\end{aligned}
$$

On the other hand, when no wave propagation is possible in the upper layer (i.e., when $k^{2}>l_{2}^{2}$ ), the same coefficients may be written

$$
\begin{aligned}
a_{1}= & \frac{1}{2} i U k \hat{h} \\
& \times \frac{e^{|k| H}\left(|k| H+n_{2} H-g^{\prime} H / U^{2}\right)}{|k| H \cosh (|k| H)+\left(n_{2} H-g^{\prime} H / U^{2}\right) \sinh (|k| H)},
\end{aligned}
$$

$$
\begin{aligned}
b_{1}= & \frac{1}{2} i U k \hat{h} \\
& \times \frac{e^{-|k| H}\left(|k| H-n_{2} H+g^{\prime} H / U^{2}\right)}{|k| H \cosh (|k| H)+\left(n_{2} H-g^{\prime} H / U^{2}\right) \sinh (|k| H)} .
\end{aligned}
$$

For the purpose of calculating the surface drag exerted on the mountain ridge, the pressure perturbation associated with the waves $p$ needs to be determined. It can be shown that, within each layer, the Fourier transform of this quantity is given in general by

$$
\hat{p}=i \frac{\rho_{0}}{k}[(d U / d z) \hat{w}-U(d \hat{w} / d z)],
$$

where $\rho_{0}$ is a reference density (assumed to be constant). In fact, for the flow considered here $d U / d z=0$, so only the second term in the parentheses in (13) is nonzero, and the Fourier transform of the pressure perturbation 
in the lower layer $\hat{p}_{1}$ evaluated at the surface can be written

$$
\hat{p}_{1}(z=0)=i \rho_{0} U \operatorname{sgn}(k)\left(a_{1}-b_{1}\right) .
$$

The surface drag force directed across the ridge per unit length in the cross-flow direction is defined as (Teixeira et al. 2013)

$$
\begin{aligned}
& D=\int_{-\infty}^{+\infty} p_{1}(z=0) \frac{\partial h}{\partial x} d x \quad \text { or } \\
& D=4 \pi \operatorname{Im}\left[\int_{0}^{+\infty} k \hat{p}_{1}(z=0) \hat{h}^{*} d k\right],
\end{aligned}
$$

where $p_{1}$ is the pressure perturbation in the lower layer, $h(x)$ is the surface elevation, the asterisk denotes complex conjugate and "Im" denotes imaginary part. For the current purpose, and as done in a number of previous studies, the ridge is assumed to be symmetric and bell shaped, defined as

$$
h=\frac{h_{0}}{1+(x / a)^{2}} \Rightarrow \hat{h}=\frac{h_{0} a}{2} e^{-a|k|},
$$

where $h_{0}$ is its maximum elevation and $a$ is its halfwidth. It turns out that the total drag can be split into two parts: $D=D_{I}+D_{L}, D_{I}$ being associated with internal waves propagating in the upper-atmospheric layer $z>H$, and $D_{L}$ with lee waves trapped at the inversion existing at $z=H$. The term $D_{I}$ receives contributions from low wavenumbers satisfying $k^{2}<l_{2}^{2}$, while $D_{L}$ receives contributions from high wavenumbers, for which $k^{2}>l_{2}^{2}$ (corresponding to waves that are evanescent in the upper layer). Using (15), (14), and (9)-(12), each of these drag components is found to be

$$
\begin{aligned}
& D_{I}=4 \pi \rho_{0} U^{2} \int_{0}^{l_{2}} k^{2}|\hat{h}|^{2} \frac{\left(m_{2} H\right)(k H)}{\left[k H \cosh (k H)-\left(g^{\prime} H / U^{2}\right) \sinh (k H)\right]^{2}+\left(m_{2} H\right)^{2} \sinh ^{2}(k H)} d k, \\
& D_{L}=4 \pi \rho_{0} U^{2} \operatorname{Im}\left[\int_{l_{2}}^{+\infty} k^{2}|\hat{h}|^{2} \frac{k H \sinh (k H)+\left(n_{2} H-g^{\prime} H / U^{2}\right) \cosh (k H)}{k H \cosh (k H)+\left(n_{2} H-g^{\prime} H / U^{2}\right) \sinh (k H)} d k\right],
\end{aligned}
$$

where the imaginary part of the integral was taken to obtain (17) from (15). This partition of the drag bears some resemblance to that presented by Teixeira et al. (2013), with the difference that $D_{L}$ is associated here with interfacial waves instead of internal waves propagating in a layer near the surface. If the upper-atmospheric layer at $z>H$ extended down to the surface $z=0$ and the flow was hydrostatic, the drag would take the wellknown form

$$
D_{0}=\frac{\pi}{4} \rho_{0} N_{2} U h_{0}^{2}=\frac{\pi}{4} \rho_{0} U^{2} l_{2} h_{0}^{2}
$$

This will be the reference value used to normalize both $D_{I}$ and $D_{L}$. If $k, m_{2}$, and $n_{2}$ are similarly normalized using $H$ as $k^{\prime}=k H, m_{2}^{\prime}=m_{2} H$ and $n_{2}^{\prime}=n_{2} H$, and if a Froude number is defined as $\mathrm{Fr}=U /\left(g^{\prime} H\right)^{1 / 2}$, (17) takes the form

$$
\begin{aligned}
\frac{D_{I}}{D_{0}}= & 4\left(\frac{a}{H}\right)^{2} \frac{1}{l_{2} H} \\
& \times \int_{0}^{l_{2} H} \frac{k^{\prime 3} m_{2}^{\prime} e^{-2 k^{\prime}(a / H)}}{\left(k^{\prime} \cosh k^{\prime}-\mathrm{Fr}^{-2} \sinh k^{\prime}\right)^{2}+m_{2}^{\prime 2} \sinh ^{2} k^{\prime}} d k^{\prime}
\end{aligned}
$$

where (16) has also been used. Note that Fr is different from what is sometimes called "Froude number" (Reinecke and Durran 2008): $\left(l_{2} h_{0}\right)^{-1}$, which is a measure of flow nonlinearity. The integral in (20) must be calculated numerically, and here a Gauss-Legendre quadrature algorithm will be employed for that purpose. If $D_{L}$ is made dimensionless in a similar way, and (16) is also taken into account, (18) becomes

$$
\frac{D_{L}}{D_{0}}=4\left(\frac{a}{H}\right)^{2} \frac{1}{l_{2} H} \operatorname{Im}\left[\int_{l_{2} H}^{+\infty} k^{\prime 2} e^{-2 k^{\prime}(a / H)} \frac{k^{\prime} \sinh k^{\prime}+\left(n_{2}^{\prime}-\mathrm{Fr}^{-2}\right) \cosh k^{\prime}}{k^{\prime} \cosh k^{\prime}+\left(n_{2}^{\prime}-\mathrm{Fr}^{-2}\right) \sinh k^{\prime}} d k^{\prime}\right]
$$

As in the trapped-lee-wave drag expression of Teixeira et al. (2013), the integrand in (21) is real, but, unlike that in (20), may have singularities along the real axis that contribute to the imaginary part of the associated integral. 
Such singularities correspond to trapped-lee-wave modes. Trapped lee waves may occur for a value of $k^{\prime}=k_{L}^{\prime}$ for which the denominator in the integrand of (21) is zero; that is,

$$
\tanh \left(k_{L}^{\prime}\right)=\frac{k_{L}^{\prime}}{\operatorname{Fr}^{-2}-n_{2}^{\prime}\left(k_{L}^{\prime}\right)}
$$

as found by Vosper (2004). In fact, (22) may also be written

$$
\mathrm{Fr}^{-2}=\left[k_{L}^{\prime 2}-\left(l_{2} H\right)^{2}\right]^{1 / 2}+\frac{k_{L}^{\prime}}{\tanh \left(k_{L}^{\prime}\right)},
$$

and since $\mathrm{Fr}^{-2}$ is thus a monotonically increasing function of $k_{L}^{\prime}$, only either one trapped-lee-wave mode, or none, will exist. This contrasts with the situation treated by Scorer (1949) or Teixeira et al. (2013), where a potentially infinite number of trapped-lee-wave modes within a layer is possible. Additionally, since $k_{L}^{\prime} \geq l_{2} H$ is a necessary condition for (23) to have a real root, a trapped-lee-wave mode only exists if

$$
\operatorname{Fr}^{-2} \geq \frac{l_{2} H}{\tanh \left(l_{2} H\right)}
$$

which further means that trapped lee waves only occur for $\mathrm{Fr} \leq 1$-more specifically for $\mathrm{Fr} \leq\left(l_{2} H\right)^{-1 / 2}$, since $0 \leq$ $\tanh \left(l_{2} H\right) \leq 1$. On the other hand, (22) also implies that $k_{L}^{\prime}<\mathrm{Fr}^{-2}$. So, when trapped lee waves do exist, the resonant wavenumber $k_{L}^{\prime}$ only needs to be sought within the interval $\left[\left(l_{2} H\right), \mathrm{Fr}^{-2}\right]$. This procedure must be carried out numerically, using (23), and is implemented here using an iterative Newton-Raphson algorithm, with an imposed relative precision of $10^{-6}$ for $k_{L}^{\prime}$ (estimated by the difference between two successive iterations).

When (24) is not satisfied, $D_{L}=0$, whereas when it is satisfied, the integral in (21) must be evaluated by contour integration, taking into account the contribution from the singularity at $k_{L}^{\prime}$. As in Teixeira et al. (2013), it can be shown that also here the integration path must pass above the singularity, yielding

$$
\frac{D_{L}}{D_{0}}=4 \pi\left(\frac{a}{H}\right)^{2} \frac{1}{l_{2} H} k_{L}^{\prime 2} e^{-2 k_{L}^{\prime}(a / H)} \frac{\left[\mathrm{Fr}^{-2}-n_{2}^{\prime}\left(k_{L}^{\prime}\right)\right]^{2}-k_{L}^{\prime 2}}{k_{L}^{\prime 2}\left[1+n_{2}^{\prime}\left(k_{L}^{\prime}\right)^{-1}\right]+\left[1+n_{2}^{\prime}\left(k_{L}^{\prime}\right)-\mathrm{Fr}^{-2}\right]\left[\mathrm{Fr}^{-2}-n_{2}^{\prime}\left(k_{L}^{\prime}\right)\right]}
$$

This is the main result of the present paper. Unlike the corresponding expression in Teixeira et al. (2013) for waves trapped within a layer, (25) does not contain a sum, because there is at most only one trapped-leewave mode. Additionally, while it is not obvious that $D_{L} / D_{0}$ is nonnegative (the sign depends on the selected integration path), that is actually the case, as makes sense physically. Equation (25), or rather its dimensional version, does not reduce to (2.2.25) of Baines (1995) for surface wave drag when the stratification of the upper layer approaches zero $\left(l_{2} H \rightarrow 0\right)$ because Baines's equation assumes that the density difference between the two layers is large (in fact in his calculations the upper layer does not exist and the lower layer is bounded above by a free surface). This contrasts with the present situation, where the density in the pressure continuity condition that originates (8) is assumed to be constant across the interface, which is consistent with (13).

As for the corresponding drag expression in Teixeira et al. (2013), (25) was confirmed alternatively using the general trapped-lee-wave drag formula obtained by Smith (1976) as a generalization to an unbounded atmosphere of that originally derived by Bretherton (1969); namely,

$$
D_{L}=2 \pi^{2} \rho_{0} U^{2}\left|\hat{h}\left(k_{L}\right)\right|^{2} \frac{\left|\frac{d \hat{w}}{d z}\left(k_{L}, z=0\right)\right|^{2}}{\int_{0}^{+\infty}\left|\hat{w}\left(k_{L}, z\right)\right|^{2} d z},
$$

when the wave solutions in the two layers were inserted into (26) and the integral in the denominator was calculated analytically. This provides a fairly strong check on the correctness of the above analysis.

In (20) and (25), $D_{I} / D_{0}$ and $D_{L} / D_{0}$ depend on the dimensionless parameters $l_{2} H, \mathrm{Fr}$, and $a / H\left[k_{L}^{\prime}\right.$, which appears in (25) and is given implicitly by (23), depends on $l_{2} \mathrm{H}$ and $\mathrm{Fr}$, but not on $\left.\mathrm{a} / \mathrm{H}\right]$. As noted by Teixeira et al. (2013), as long as three independent input parameters are preserved, the above quantities may be combined as convenient. Here, following Vosper (2004), $l_{2} \mathrm{H}, \mathrm{Fr}$, and $l_{2} a$ will be adopted as the key input parameters. In what follows, the behavior of $D_{I} / D_{0}$ and $D_{L} / D_{0}$ will be explored as a function of $l_{2} \mathrm{H}$ and $\mathrm{Fr}$ for selected values of $l_{2} a$. 

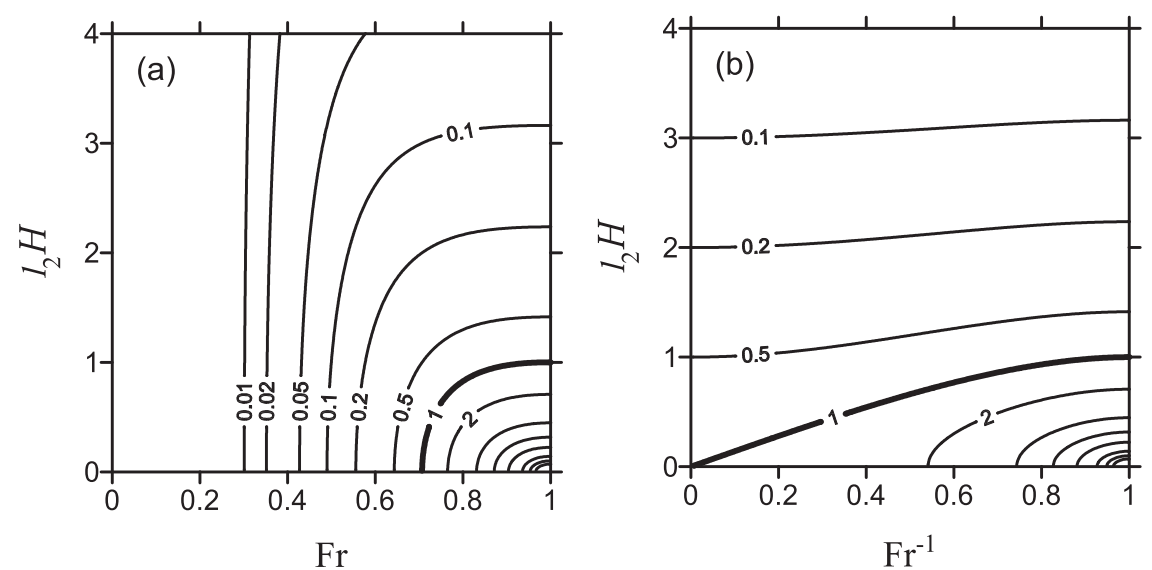

FIG. 1. Normalized propagating-wave drag (equal to the total drag) as a function of Fr and $l_{2} H$ in exactly hydrostatic conditions $\left(l_{2} a \rightarrow \infty\right)$. Contours at $0.01,0.02,0.05,0.1,0.2,0.5$, and so on. (a) $0<\operatorname{Fr}<1$; (b) $1<\operatorname{Fr}<\infty$.

In the hydrostatic approximation (i.e., for $l_{2} a \rightarrow+\infty$ ), it can be shown that (20) reduces to

$$
\frac{D_{I}}{D_{0}}=\frac{1}{\left(1-\mathrm{Fr}^{-2}\right)^{2}+\left(l_{2} H\right)^{2}},
$$

and the trapped-lee-wave drag, which only exists in nonhydrostatic conditions, necessarily vanishes. Equation (27) shows that the normalized drag is independent of the orography shape (cf. Teixeira et al. 2008), and decreases as $l_{2} H$ increases. For all $\mathrm{Fr} \neq 0$, the total drag $D / D_{0}$ is nonzero, and the drag behavior is nonsingular at Fr $=1$, unless $l_{2} H=0$ (the case where the upper layer is neutrally stratified). The drag attains a maximum at Fr $=1$ of magnitude $1 /\left(l_{2} H\right)^{2}$, which obviously should become invalid for sufficiently low values of $l_{2} H$, when this maximum becomes much larger than 1 . Under those circumstances, either friction, or nonlinearity, or both effects should become important [see Teixeira et al. (2012), where frictional effects were included in a simplified way]. The fact that even in the neutrally stratified case $\left(l_{2}=0\right.$ or $\left.l_{2} H=0\right) D / D_{0}=D_{I} / D_{0}$ is nonzero for Fr $\neq 1$ is an artifact of the way in which the drag is normalized using $D_{0}$, since this latter quantity is itself zero in that case.

\section{Results: Drag behavior}

First, it is instructive to consider the behavior of the drag in exactly hydrostatic conditions, when it depends only on $l_{2} H$ and Fr. Figure 1 shows $D_{I} / D_{0}$ for $l_{2} a \rightarrow \infty$ (which is equal to $D / D_{0}$ ) given by (27) as a function of these two parameters. Figure $1 \mathrm{a}$ is for $0<\mathrm{Fr}<1$ and Fig. $1 \mathrm{~b}$ for $1<\operatorname{Fr}<\infty$, so as to cover the complete possible range of variation of Fr. As mentioned above,
$D / D_{0}$ decreases as $l_{2} H$ increases, since this parameter quantifies the depth of the layer, near the surface, where the gravity waves are evanescent. As the layer aloft where the gravity waves propagate vertically is lifted, its effect on the surface pressure, which determines the surface drag, is attenuated. $D / D_{0}$ approaches zero as Fr $\rightarrow 0$, and $D / D_{0} \rightarrow 1$ as $\mathrm{Fr} \rightarrow \infty$ when $l_{2} H=0$, as makes sense physically, since this last limit corresponds to a vanishing inversion strength and thickness of the neutral layer. At $\mathrm{Fr}=1$ and $l_{2} H=0$ the drag has a singular behavior, tending to infinity, as shown by (27). When $l_{2} H>0$, the drag goes from zero at $\mathrm{Fr} \approx 0$ to a value lower than one at $\mathrm{Fr} \approx \infty$, attaining a finite maximum at $\mathrm{Fr}=1$. For $l_{2} H=1$ and $\mathrm{Fr}=1$, for example, the increase of $D / D_{0}$ due to the effect of the inversion (encapsulated in Fr) compensates its decrease due to $l_{2} \mathrm{H}$, and the drag is equal to that valid for a single stratified layer extending down to the surface.

Lee waves trapped at the inversion only arise for finite values of $l_{2} a$, because this is necessary for downstream propagation of the waves to be possible. However, the wavelength of these waves does not depend on $l_{2} a$, but only on $l_{2} \mathrm{H}$ and Fr [note that Teixeira et al. (2013) showed that the wavelength of lee waves trapped within a layer did not depend on $l_{1} a-$ a related measure of nonhydrostatic effects]. Figure 2 shows the wavelength of trapped lee waves $\lambda_{L}$ normalized by the hydrostatic vertical wavelength of propagating waves in the upper layer, $\lambda_{L} l_{2} /(2 \pi)=l_{2} / k_{L}$. No trapped lee waves are allowed to exist to the right of the thick solid curve, which corresponds to (24) with the inequality sign replaced by an equality sign. At this curve, the normalized wavelength takes a value of one. As either $l_{2} H$ or Fr decrease, the wavelength decreases toward zero. Unlike in Teixeira et al. (2013), there is no lower bound on the value of the 


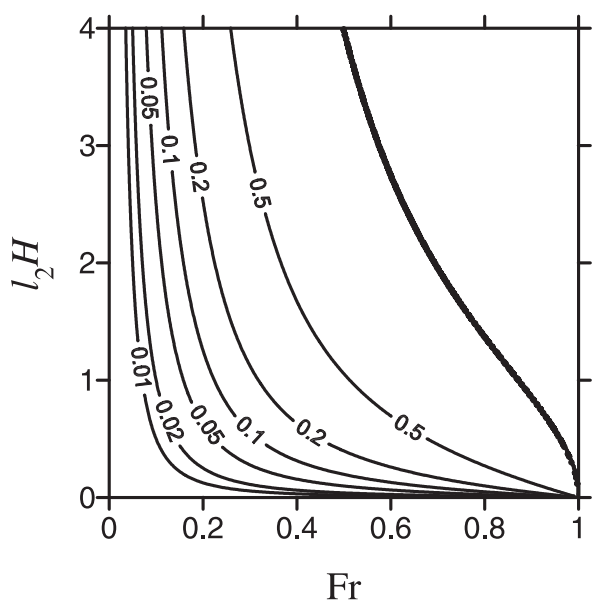

FIG. 2. Normalized wavelength of the trapped lee waves $\lambda_{L} l_{2} /(2 \pi)=l_{2} / k_{L}$ as a function of Fr and $l_{2} H$. This quantity is independent of $l_{2} a$. Contours at 0.01, 0.02, 0.05, 0.1, 0.2, 0.5, and so on. Thick contour is 1 . No trapped lee waves are allowed to exist to the right of the thick curve.

wavelength, since the upper bound of $k$ in the integral in (21) is infinity. This means that very short trapped lee waves are possible, although their amplitude and impact on the drag are relatively small, as will be seen next.

The drag is now calculated for more practically relevant situations than considered in Fig. 1, with finite $l_{2} a$. Figures 3-7 present the normalized drag as a function of $l_{2} \mathrm{H}$ and $\mathrm{Fr}$ for $l_{2} a=5,2,1,0.5$, and 0.2 , respectively. These values were deemed to be the best for illustrating the range of variation of the trapped-lee-wave drag, although, admittedly, the last two values would correspond to rather narrow (and thus, in reality, necessarily low) obstacles. Taking typical values of $N_{2}=0.01 \mathrm{~s}^{-1}$ and $U=10 \mathrm{~m} \mathrm{~s}^{-1}$, this yields $l_{2}=N_{2} / U=10^{-3} \mathrm{~m}^{-1}$, so that using the previous $l_{2} a$ values, $a=5000,2000,1000$, 500 , and $200 \mathrm{~m}$, respectively. However, if $l_{2}$ was assumed to be lower, the estimated $a$ would be larger.

Figures 3a, 4a, 5a, 6a, and 7a present the propagatingwave drag $D_{I} / D_{0}$ for $0<\mathrm{Fr}<1$; Figs. $3 \mathrm{~b}, 4 \mathrm{~b}, 5 \mathrm{~b}, 6 \mathrm{~b}$, and $7 \mathrm{~b}$ present the trapped-lee-wave $\operatorname{drag} D_{L} / D_{0}$ for the same range of Fr (which is the only one where this component of the drag is nonzero). Figures $3 c, 4 c, 5 c, 6 c$, and $7 \mathrm{c}$ present the total drag, defined as $D / D_{0}=D_{I} / D_{0}+$ $D_{L} / D_{0}$, and Figs. $3 \mathrm{~d}, 4 \mathrm{~d}, 5 \mathrm{~d}, 6 \mathrm{~d}$, and $7 \mathrm{~d}$ present $D_{I} / D_{0}$ for $1<\mathrm{Fr}<\infty$ (which is equal to the total drag in this parameter range). In Figs. 3a-c, $4 \mathrm{a}-\mathrm{c}, 5 \mathrm{a}-\mathrm{c}, 6 \mathrm{a}-\mathrm{c}$, and $7 \mathrm{a}-\mathrm{c}$, the region of parameter space where trapped lee waves are allowed to exist is to the left of the dashed curve (which corresponds to the thick solid curve in Fig. 2).

As one moves from Figs. 3a,d to Figs. 7a,d, $D_{I} / D_{0}$ generally decreases relative to the values displayed in
Fig. 1, owing to nonhydrostatic effects (which lead to wave dispersion). This is especially visible in the value taken by $D_{I} / D_{0}$ for $l_{2} H=0$ and $\mathrm{Fr} \rightarrow \infty$, which is already somewhat lower than 1 for $l_{2} a=5$ (Fig. 3d), still between 0.5 and 1 for $l_{2} a=2$ (Fig. $4 \mathrm{~d}$ ), between 0.2 and 0.5 for $l_{2} a=1$ (Fig. 5d), between 0.1 and 0.2 for $l_{2} a=0.5$ (Fig. 6d), and between 0.02 and 0.05 for $l_{2} a=0.2$ (Fig. 7d). The singular behavior of $D_{I} / D_{0}$ for $l_{2} H=0$ and $\mathrm{Fr}=1$ appears to be preserved for finite $l_{2} a$. A curious feature of the $D_{I} / D_{0}$ fields displayed in Figs. 3a, 4a, 5a, $6 \mathrm{a}$, and 7a, especially salient for the lowest values of $l_{2} a$ (e.g., $l_{2} a=1,0.5$, and 0.2 ), is the sharp angle made by the contours as they pass across the dashed curve, especially for low values of $l_{2} H$. This sharp angle corresponds to the existence of a gap in the $D_{I} / D_{0}$ field, which is compensated by the existence of a considerable complementary $D_{L} / D_{0}$, as will be described next.

In Figs. 3b, 4b, 5b, 6b, and 7b, it can be seen that $D_{L} / D_{0}$ is almost insignificant for $l_{2} a=5$ (Fig. 3b) but becomes nonnegligible for $l_{2} a=2$ (Fig. $4 \mathrm{~b}$ ), taking values $O(1)$ for sufficiently low $l_{2} H$ and $\mathrm{Fr} \approx 1$. The oblique region of parameter space where $D_{L} / D_{0}$ is nonzero is located to the left and relatively close to the dashed curve delimiting the occurrence of trapped lee waves, with an absolute maximum for $l_{2} H=0$ and $\mathrm{Fr}=1$, where the drag is singular. This means that the trapped lee waves that produce most of the drag are not very short, having a normalized wavelength that is generally larger than 0.2 and mostly larger than 0.5 . When $l_{2} a=1$ (Fig. 5b), the cases with significant trapped-lee-wave drag spread to a wider region of parameter space, with $D_{L} / D_{0}$ attaining values larger than 1 for realistic values of the input parameters, such as $l_{2} \mathrm{H}=0.5$ and $\mathrm{Fr}=0.8$ (corresponding, for $\theta_{0}=283 \mathrm{~K}$ and the values of $N_{2}$ and $U$ estimated above, to $H=500 \mathrm{~m}$ and $\Delta \theta=9 \mathrm{~K}$ ). For this value of $l_{2} a, D_{L} / D_{0}$ is clearly of a magnitude comparable to that of $D_{I} / D_{0}$, assuming therefore considerable dynamical significance. When $l_{2} a=0.5$ (Fig. 6b), the trapped-lee-wave drag is still highly relevant, increasing its importance relative to the propagating-wave drag, because the latter decreases. The region of parameter space with significant $D_{L} / D_{0}$ moves to lower values of both $l_{2} \mathrm{H}$ and Fr. For $l_{2} a=0.2$ (Fig. 7b), the trappedlee-wave drag starts to decrease significantly, like the propagating-wave drag, and in the representation adopted here its pattern continues to flatten vertically, being confined to lower values of $l_{2} H$.

Figures $3 c, 4 c, 5 c, 6 c$, and $7 c$, displaying the total drag, show that, as in Teixeira et al. (2013), the trapped-leewave drag takes in the present problem a complementary role to the propagating-wave drag, filling the gaps existing in the field of the latter. Hence, while the sharp angles in the $D_{I} / D_{0}$ contours are more pronounced the 

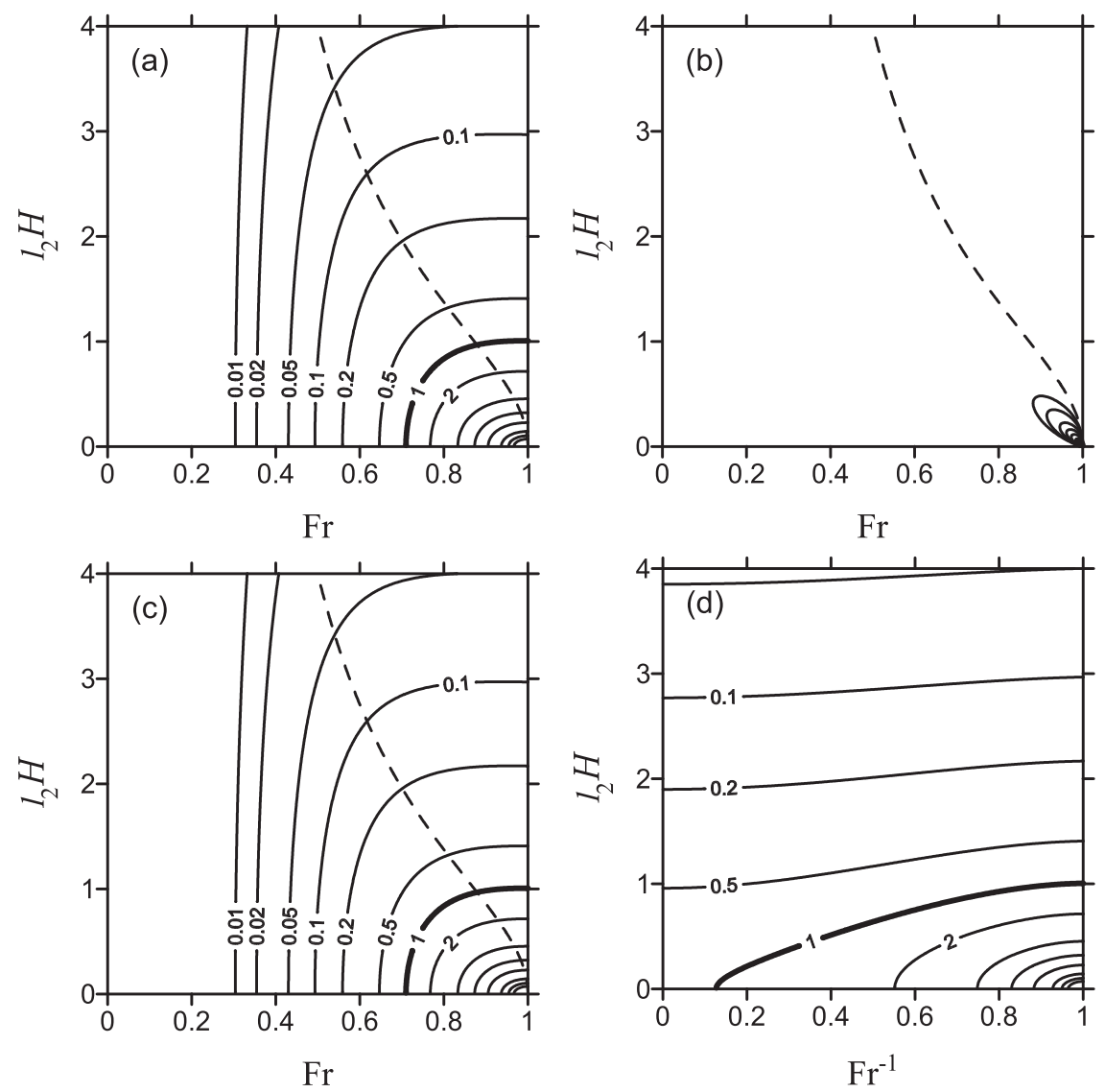

FIG. 3. Normalized drag as a function of $\mathrm{Fr}$ and $l_{2} \mathrm{H}$ for $l_{2} a=5$. Contours at 0.01, 0.02, 0.05, $0.1,0.2,0.5$, and so on. (a) Propagating-wave drag, (b) trapped-lee-wave drag, and (c) total drag $D / D_{0}=D_{I} / D_{0}+D_{L} / D_{0}$ for $0<\mathrm{Fr}<1$, and (d) propagating-wave drag (equal to the total drag) for $1<\operatorname{Fr}<\infty$. Trapped lee waves only exist to the left of the dashed curve.

larger $D_{L} / D_{0}$ is, these features are totally suppressed in the $D / D_{0}$ field, which shows that $D_{I} / D_{0}$ and $D_{L} / D_{0}$ complement each other. As $l_{2} a$ decreases, the region of parameter space where $D / D_{0}$ is $O(1)$ moves to lower values of $l_{2} \mathrm{H}$ and also to lower Fr. Clearly, the regions of parameter space where $\mathrm{Fr} \ll 1$ or $l_{2} H \ll 1$ are not very relevant meteorologically, since they correspond to situations with unrealistically low or unrealistically strong inversions. Therefore, the behavior of the propagatingwave drag and of the trapped-lee-wave drag will be analyzed in the next section as a function of Fr mostly for $l_{2} H=0.5-$ a reasonable value that is within the range of values considered by Vosper (2004) for this parameterand leads to substantial resonant enhancement of both $D_{I} / D_{0}$ and $D_{L} / D_{0}$.

Meanwhile, the previous results may help to shed some light on why, in the studies of Grubišić and Stiperski (2009) and Stiperski and Grubišić (2011), the inclusion of an inversion in the static stability profile was found to have a relatively minor impact on the behavior of trapped lee waves. While these studies were primarily focused on atmospheric profiles where the Scorer parameter smoothly decreases with height [corresponding therefore to the different wave trapping mechanism addressed by Teixeira et al. (2013)], analogies may probably still be drawn with the present model setup. A temperature inversion is such a strong perturbation to the Scorer parameter profile (approaching a Dirac delta singularity in the idealized limit) that its effects may perhaps be superposable on the remainder. Hence, in Grubišić and Stiperski (2009) and Stiperski and Grubišić (2011), $\Delta \theta=4.84 \mathrm{~K}$, and if their values of the potential temperature $\theta_{0}=310 \mathrm{~K}$, of the incoming wind speed $U=30 \mathrm{~m} \mathrm{~s}^{-1}$, and of the Brunt-Väisälä frequency $N_{2}=$ $0.01 \mathrm{~s}^{-1}$ are taken immediately above the inversion, at $z=H=5260 \mathrm{~m}$, this yields $\mathrm{Fr}=0.95$ and $l_{2} H=1.75$. Figures 3-7 suggest that $D_{L} / D_{0}$, and thus the intensity of the associated trapped lee waves, should be quite small under these conditions for any value of $l_{2} a$, including the value consistent with the numerical simulations of 

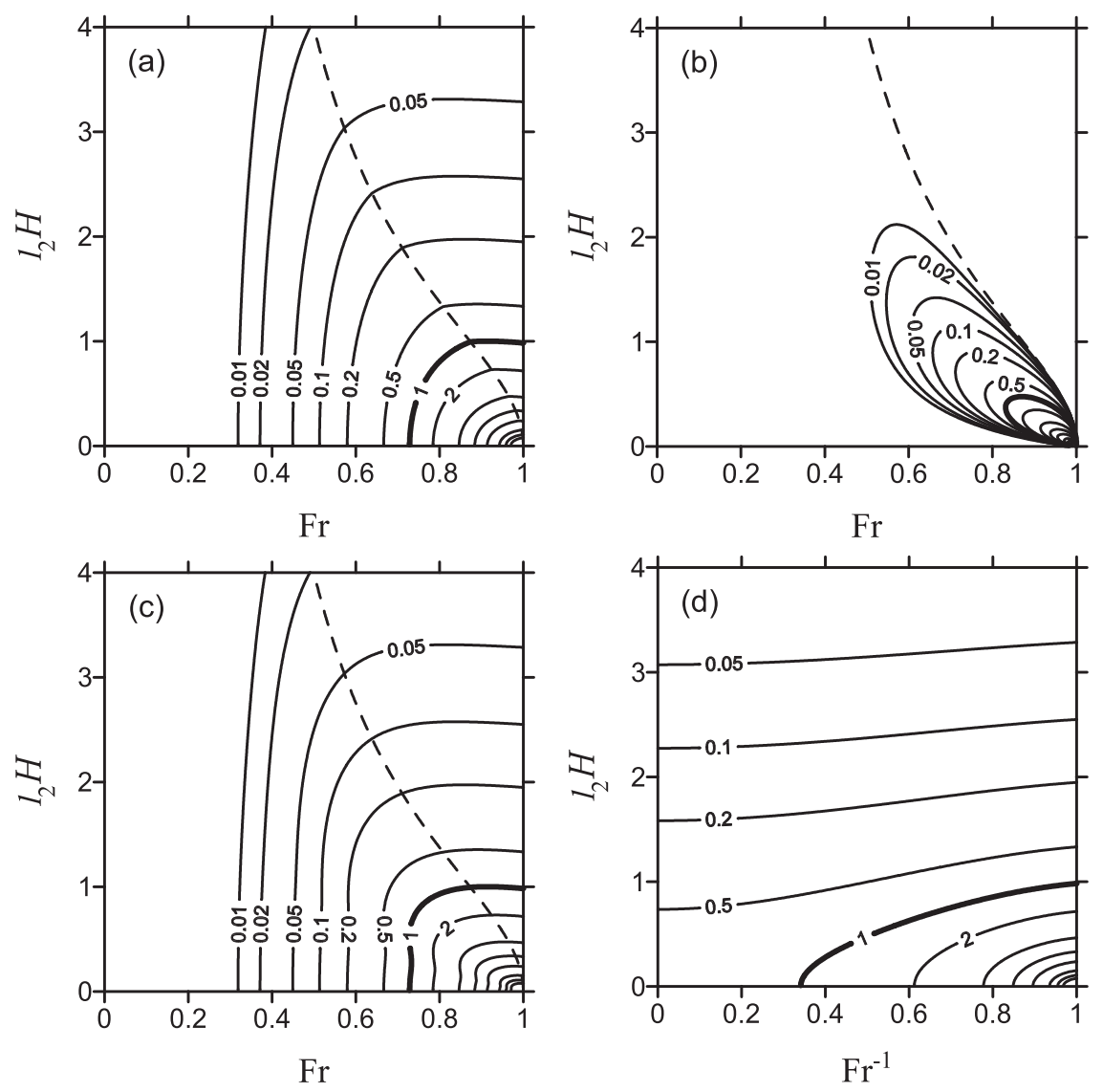

FIG. 4. As in Fig. 3, but for $l_{2} a=2$.

Grubišić and Stiperski (2009): $l_{2} a=1$.67. Although these inferences must be viewed with caution, because the atmospheric profile used by Grubišić and Stiperski (2009) and Stiperski and Grubišić (2011) differs markedly in other respects from the one assumed in the present study, it is reasonable to suggest that a lower inversion height would probably be required to have a more substantial impact on the flow dynamics than verified by those authors.

\section{a. Results for fixed $l_{2} \mathrm{H}$ and $l_{2} a$}

Figure 8 shows the normalized wavelength of trapped lee waves $\lambda_{L} l_{2} /(2 \pi)$ as a function of Fr for $l_{2} H=2,1$, and 0.5 , calculated from the same linear model as used previously (curves). For the typical value of the Scorer parameter estimated before, $l_{2}=10^{-3} \mathrm{~m}^{-1}$, this corresponds to $H=2000,1000$, and $500 \mathrm{~m}$, which are plausible inversion heights. The normalized wavelength, which as mentioned above does not depend on $l_{2} a$, increases from values near zero at $\mathrm{Fr} \approx 0$ to a value of one at an upper limit of $\mathrm{Fr}<1$, which increases as $l_{2} \mathrm{H}$ decreases, in accordance with Fig. 2. This upper limit is determined by the relation (24), which implies that $\mathrm{Fr} \leq$ 0.69 for $l_{2} H=2, \mathrm{Fr} \leq 0.87$ for $l_{2} H=1$, and $\mathrm{Fr} \leq 0.96$ for $l_{2} H=0.5$, which is in agreement with Fig. 8. Also shown in Fig. 8 as the symbols are results from simulations carried out using FLEX - a 2D nonlinear and nonhydrostatic numerical model described by Argaín et al. (2009). This model was run in inviscid and nonrotating mode, and in very nearly linear conditions $\left(l_{2} h_{0}=0.01\right.$ and $\left.h_{0} / a \leq 0.05\right)$, since the aim here is just to check the predictions from the linear model (see details of the simulations in the appendix). It can be seen that, under these assumptions, linear theory is able to predict the wavelength of trapped lee waves with great accuracyan aspect noted before by Vosper (2004).

The curves in Fig. 9 correspond to drag values calculated from the linear model, which will be analyzed first of all. The propagating-wave drag (dotted curves), trapped-lee-wave drag (dashed curves), and total drag (solid curves) are presented for $l_{2} H=0.5$ and different values of $l_{2} a$. It is worth keeping in mind that in the exactly hydrostatic limit and for this value of $l_{2} H,(27)$ predicts that $D / D_{0}$ changes from 0 at $\mathrm{Fr}=0$ to 0.8 at 

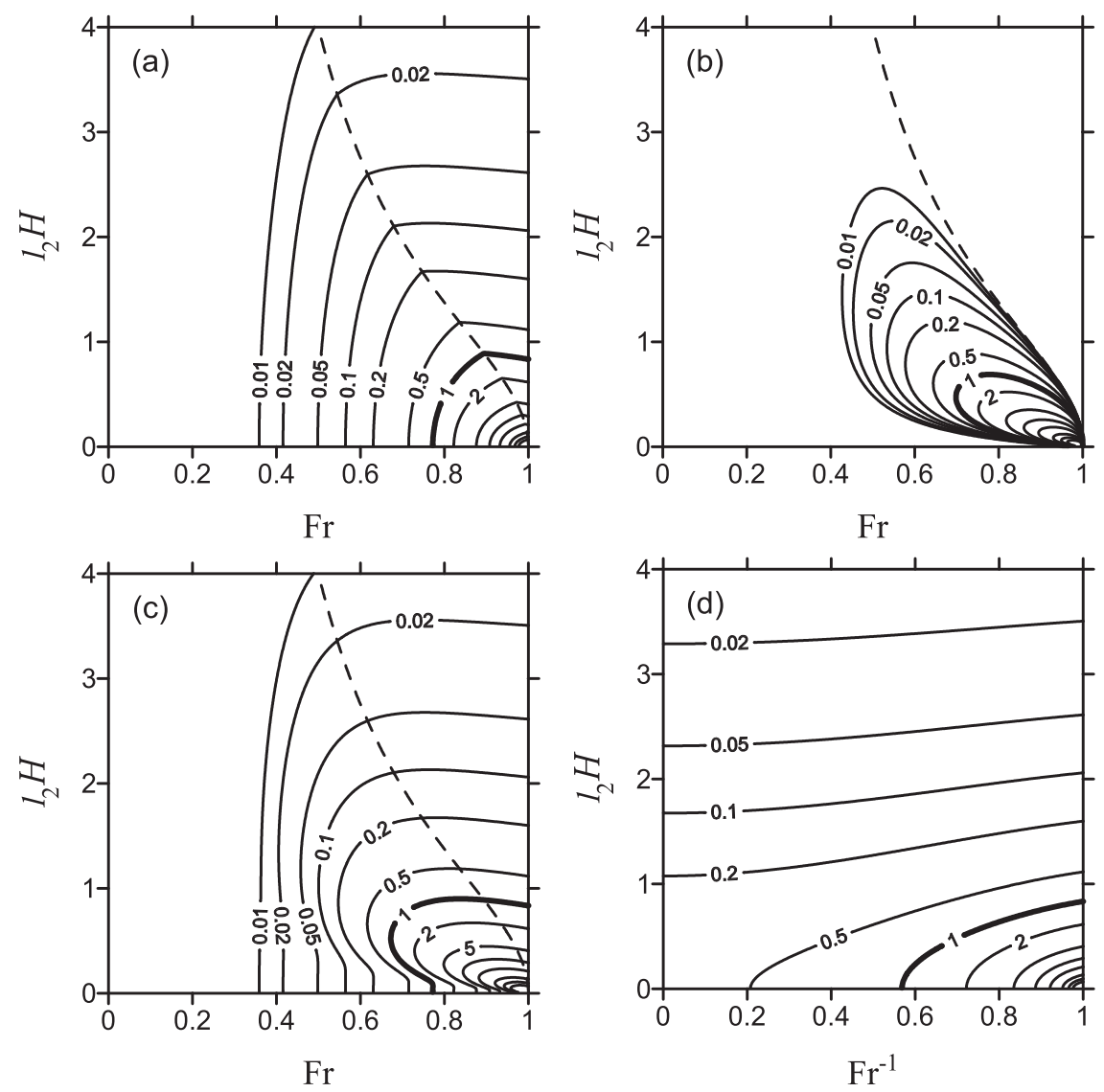

FIG. 5. As in Fig. 3, but for $l_{2} a=1$.

$\mathrm{Fr}=\infty$, attaining a maximum of $D_{I} / D_{0}=4$ at $\mathrm{Fr}=1$. For the typical values of the Scorer parameter and reference potential temperature estimated above, $l_{2}=10^{-3} \mathrm{~m}^{-1}$ and $\theta_{0}=283 \mathrm{~K}$, respectively, and $\mathrm{Fr}=1$ corresponds to $\Delta \theta=5.8 \mathrm{~K}$-a quite reasonable inversion strength.

Figure $9 \mathrm{a}$, calculated for $l_{2} a=5$, shows that the drag at high Fr tends to a value slightly smaller than $0.8(\approx 0.77)$, and the maximum at $\mathrm{Fr}=1$ is slightly larger than $4(\approx 4.15)$. This behavior overwhelmingly comes from the propagating-wave drag component, since the trappedlee-wave drag component is negligible. The fact that drag maxima in slightly nonhydrostatic conditions may exceed the hydrostatic estimate, even when the trappedlee-wave contribution is insignificant, was noted by Teixeira et al. (2013) for lee waves trapped within a layer.

In Fig. 9b, where $l_{2} a=2$ is assumed, it can be seen that the drag at high Fr has decreased to about 0.54, but the maximum of the total drag has increased to about 4.97 and shifted its location to $\mathrm{Fr} \approx 0.93$. This occurs mostly because of the propagating-wave drag, which attains a maximum of about 4.72 at $\mathrm{Fr}=0.98$. The trappedlee-wave drag now attains values $O(1)$ for Fr slightly lower than 1 and has a maximum of about 1.61 for $\mathrm{Fr} \approx 0.87$.

When $l_{2} a=1$ (Fig. 9c), the total drag at high Fr decreases to about 0.22 , and the maximum at $\mathrm{Fr} \approx 0.83$ becomes about 3.83. For this value of $l_{2} a$, the contributions of $D_{I} / D_{0}$ and $D_{L} / D_{0}$ are of comparable magnitude. The propagating-wave drag has a maximum of about 2.60 for $\mathrm{Fr} \approx 0.95$, while the trapped-lee-wave drag has a maximum of about 3.08 at $\mathrm{Fr} \approx 0.79$. It can be seen that these maxima are not coincident [as happened also in Teixeira et al. (2013)], and this is why the maximum of $D / D_{0}$ is not larger, but the $D_{L} / D_{0}$ maximum contributes to the displacement of the $D / D_{0}$ maximum toward lower values of Fr. Although the drag at high Fr is relatively modest, the drag for $\mathrm{Fr}=O(1)$ (in particular, the trapped-lee-wave component) is clearly of a magnitude relevant for gravity-wave drag parameterization.

Figure $9 \mathrm{~d}$ shows results for $l_{2} a=0.5$. The total drag at high Fr is now about 0.06 , being therefore nearly insignificant; however, the drag maximum at $\mathrm{Fr} \approx 0.81$ is about 3.28. The contribution of the trapped-lee-wave drag to this maximum has become dominant, with the propagating-wave drag attaining a maximum of about 

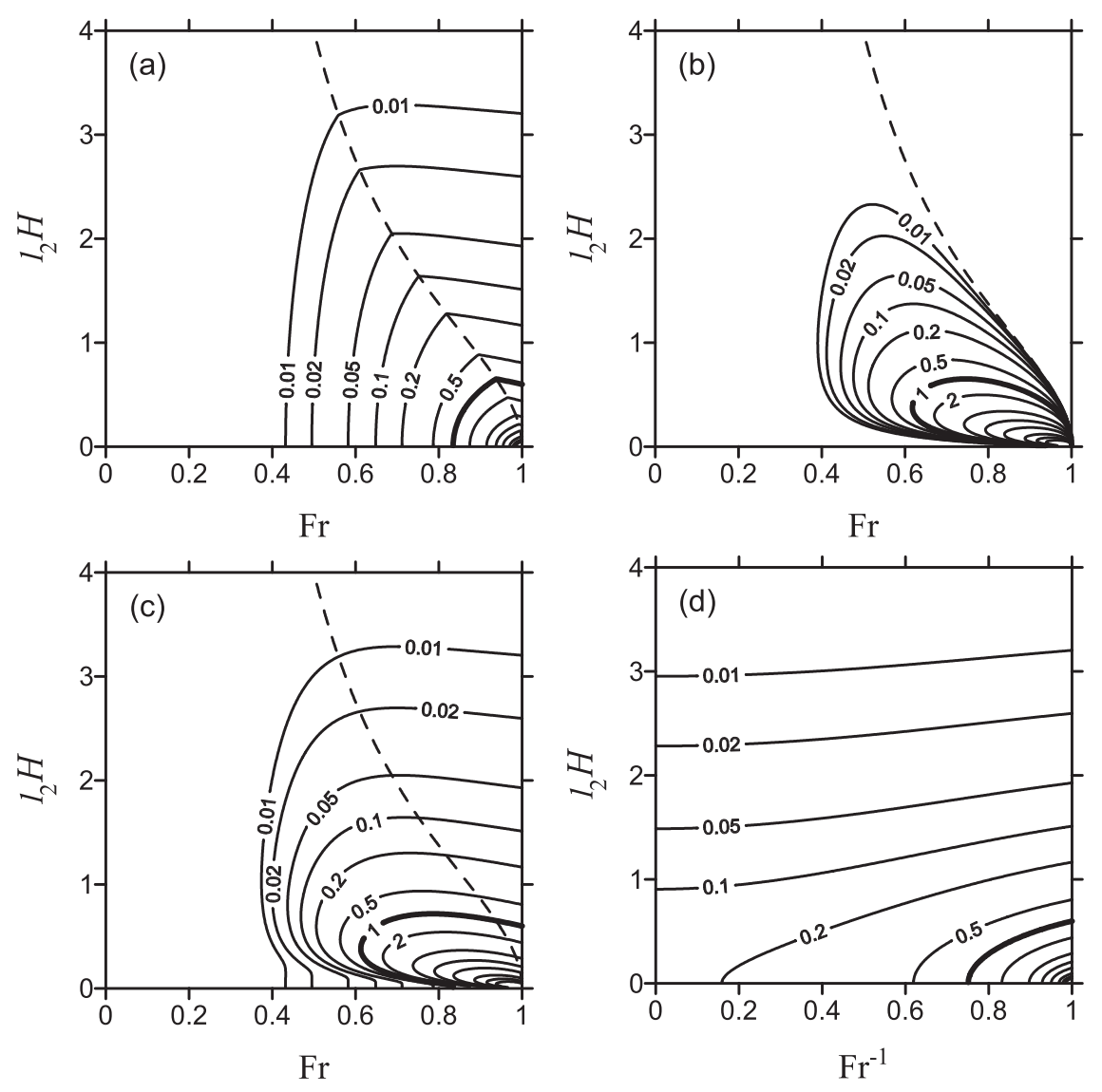

FIG. 6. As in Fig. 3, but for $l_{2} a=0.5$.

0.82 at $\mathrm{Fr} \approx 0.95$, while the maximum of the trapped-leewave drag is about 3.08 and occurs for $\mathrm{Fr} \approx 0.79$.

Finally, when $l_{2} a=0.2$ (Fig. 9e), the total drag is only about 0.01 at high $\mathrm{Fr}$ and has a maximum of about 0.40 for $\mathrm{Fr} \approx 0.66$. The drag is therefore approaching irrelevance owing to nonhydrostatic effects $\left(D / D_{0}\right.$ might only increase if $l_{2} H$ were reduced). The propagatingwave drag has a maximum of about 0.14 for $\mathrm{Fr} \approx 0.95$, and the trapped-lee-wave drag, which is by far dominant, has a maximum of about 0.39 for $\mathrm{Fr} \approx 0.66$.

In Figs. 9b-e, it is clear that the maxima of the propagating-wave drag are always centered near $\mathrm{Fr}=1$ and have sharp peaks, unlike the maxima of the trappedlee-waved drag, which are smoother (although with nonzero values spanning a narrower range of Fr) and displaced to lower values of Fr. This behavior, where $D_{L} / D_{0}$ complements $D_{I} / D_{0}$, explains the smoothness of the total drag curves, and the shift of their maxima to progressively lower values of $\mathrm{Fr}$ as $l_{2} a$ decreases. These characteristics, with some differences, parallel those found by Teixeira et al. (2013) for the drag associated with lee waves trapped in a layer. As in that study, also here it is found that the trapped-lee-wave drag can be comparable to the propagating-wave drag under realistic circumstances, and that owing to resonant amplification both drag components may significantly exceed the linear hydrostatic one-layer value $D_{0}$. These factors make the mountainwave drag remain considerably larger as the flow becomes more nonhydrostatic than would be expected based on single-layer estimates of that force.

Also shown in Figs. 9a-e are symbols corresponding to the drag predicted by numerical simulations using the FLEX model when a steady state is attained for this quantity (see details in the appendix). As in Teixeira et al. (2013), the total normalized drag is presented for all cases. Despite a slight underestimation of the total drag maxima, and some other minor differences, the general magnitude, shape, and location of the maxima are in good agreement with the linear calculations described before. In particular, for $l_{2} a=2$ and especially for $l_{2} a=1, l_{2} a=0.5$, and $l_{2} a=0.2$, both the propagatingwave component and the trapped-lee-wave component of the drag must be taken into account for this agreement to hold. This supports the soundness of the calculations carried out in section 2, and highlights the importance of these two complementary drag components. 

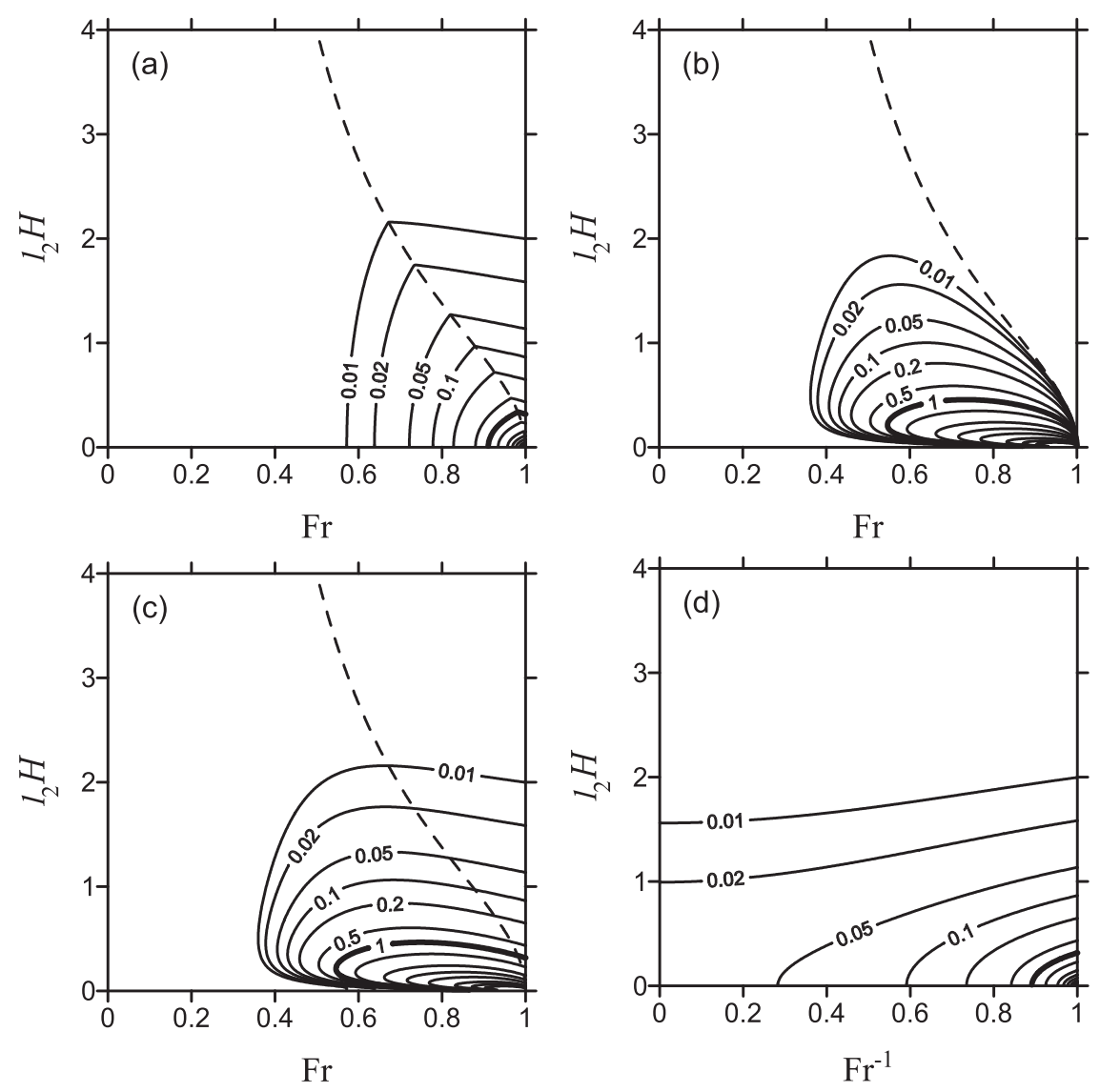

FIG. 7. As in Fig. 3, but for $l_{2} a=0.2$.

The way in which nonlinearity limits the usefulness of the above calculations may only be evaluated through additional numerical simulations. However, it should be stressed that even a reliable linear estimate for the onset of nonlinear processes [such as exists in singlelayer flow, or was obtained, for a different two-layer flow, by Teixeira and Miranda (2005)], is currently lacking for trapped-lee-wave flows. These aspects could provide a basis for future investigations.

\section{b. The regime diagram of Vosper}

Apart from their relevance to the generation of mountain-wave drag, and consequently to the parameterization of that force, trapped-lee-waves are also known to be associated with rotor circulations. The study of Vosper (2004) focused largely on the conditions favorable for the occurrence of these flow structures, where the flow reverses near the surface. In his Fig. 9, Vosper (2004) presented a regime diagram [see also Fig. 4 of Sheridan and Vosper (2006a)] where the flow structures obtained in his numerical simulations were classified into four types (no lee wave, lee wave, hydraulic jump, and lee-wave rotor) as a function of parameters Fr and $h_{0} / H$ or $\left(l_{2} H\right)^{-1}$ (in the present notation).

Since all numerical simulation results presented in Fig. 9 of Vosper (2004) use the same dimensionless mountain height $l_{2} h_{0}=0.5$ (which corresponds to only moderate nonlinearity, at least according to this singlelayer parameter), the variation of $h_{0} / H$ in that figure

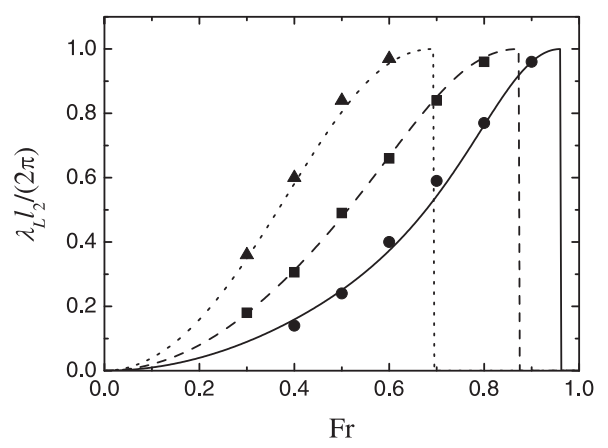

FIG. 8. Normalized wavelength of the trapped lee waves $\lambda_{L} l_{2} /(2 \pi)$ as a function of $\mathrm{Fr}$ from the linear model (curves) and from numerical simulations (symbols) for different values of $l_{2} H$. This quantity does not depend on $l_{2} a$. Solid curve and circles: $l_{2} H=0.5$; dashed curve and squares: $l_{2} H=1$; dotted curve and triangles: $l_{2} H=2$. 

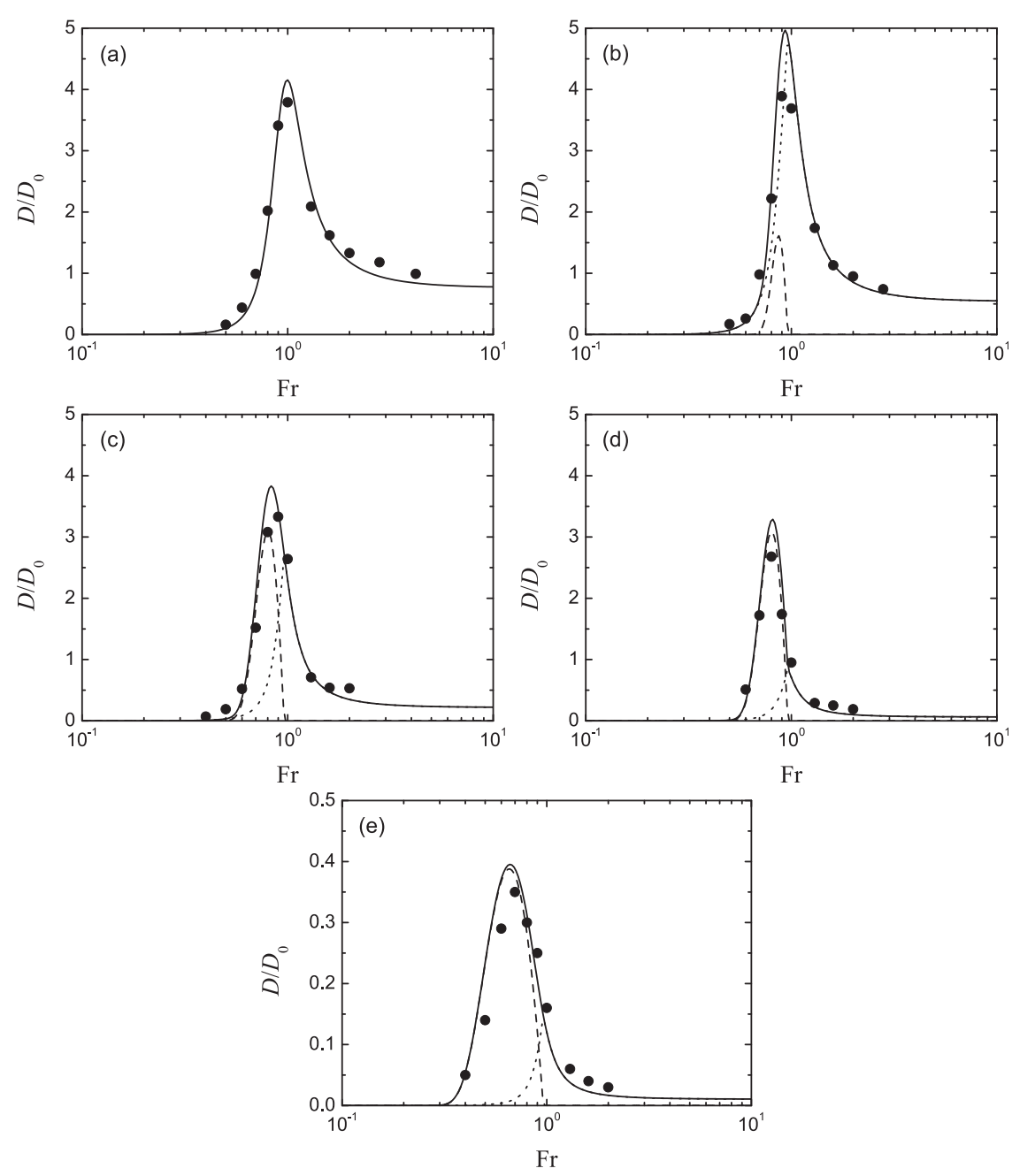

FIG. 9. Normalized drag as a function of Fr for $l_{2} H=0.5$ and different values of $l_{2} a$ from the linear model (curves) and from numerical simulations (symbols). Dotted curves: propagatingwave drag; dashed curves: trapped-lee-wave drag; solid curves and circles: total $\operatorname{drag} D / D_{0}=$ $D_{I} / D_{0}+D_{L} / D_{0}$. (a) $l_{2} a=5$, (b) $l_{2} a=2$, (c) $l_{2} a=1$, (d) $l_{2} a=0.5$, and (e) $l_{2} a=0.2$. In (a) the dotted and solid curves coincide and the dashed curve is at $D / D_{0} \approx 0$. In (e) the vertical scale was magnified for clarity.

corresponds to a proportional variation of $\left(l_{2} H\right)^{-1}$. Hence, Fig. 9 of Vosper (2004) can be directly compared with results from the linear model developed here for a similar range of $\mathrm{Fr}$ and $\left(l_{2} H\right)^{-1}$. Before this is done, however, it is necessary to decide what dimensionless mountain width to adopt in the linear calculations (the inclusion of friction or nonlinearity are beyond the scope of this study). Vosper (2004) uses a mountain that is not bell shaped but rather follows a truncated cosine function:

$$
h=\frac{1}{2} h_{0}[1+\cos (K x)], \text { for }|x| \leq \pi / K,
$$

with the mountain width defined as $L=2 \pi / K$. In the numerical simulations of Vosper (2004), the dimensionless mountain width is $l_{2} L=12.5$, apparently corresponding to nearly hydrostatic conditions. This may seem puzzling, since, according to the previous results, no trapped lee waves would be expected for that case. However, this turns out to be an artifact of the way in which $L$ is defined, as will be shown next.

The focus here is on trapped lee waves, and their intensity is determined, in the linear approximation, by the value of the Fourier transform of the orography at the resonant wavenumber. Therefore, a possible way to relate $L$ and $a$ is using the value of $k$ at which the amplitude of this Fourier transform reduces to a fraction of its value at $k=0$. At $k=1 / a$, for example, (16) shows that $\hat{h}$ reduces to $e^{-1}$ of its maximum value. Now, the Fourier transform of (28) is 

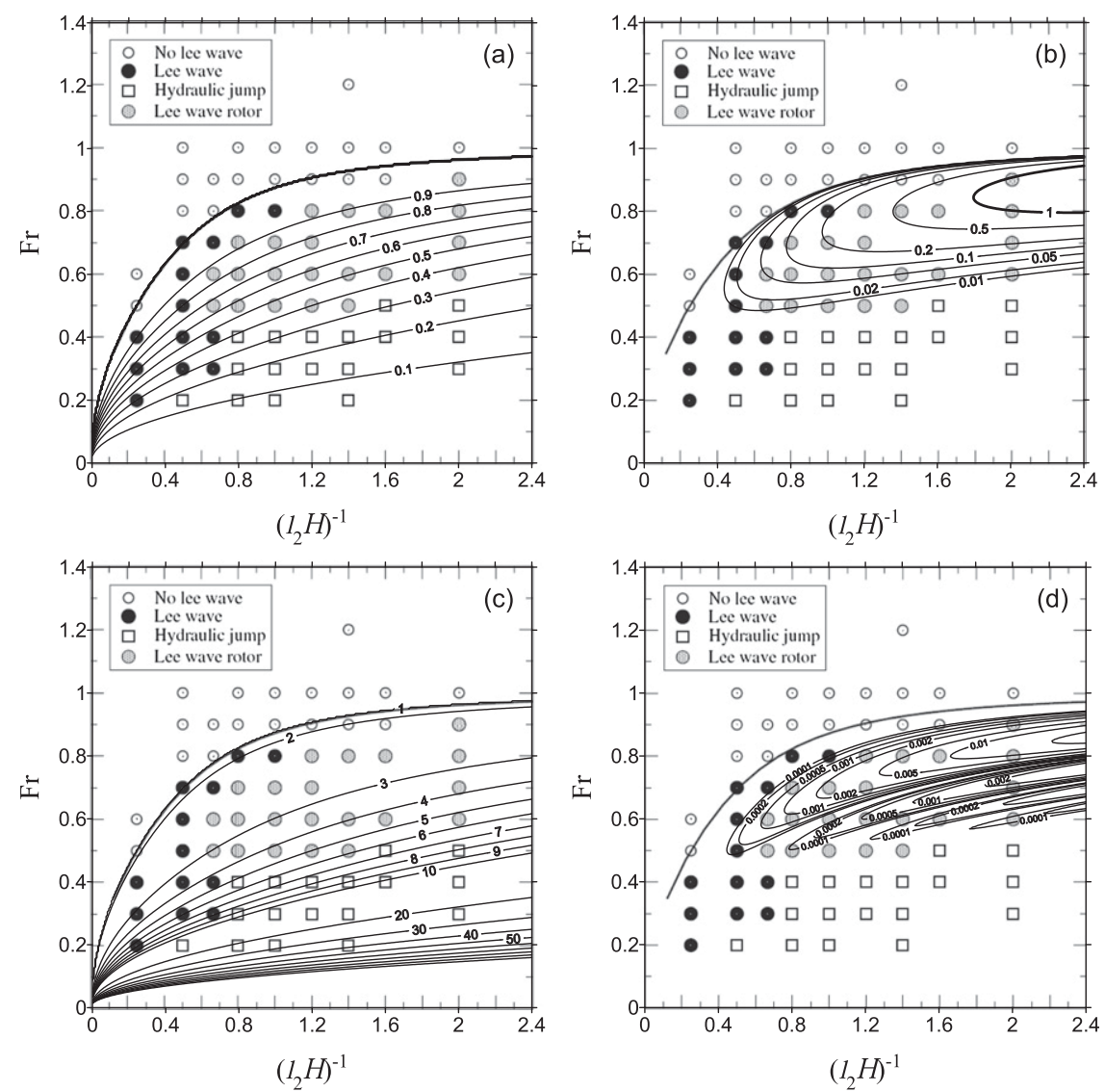

FIG. 10. Normalized wavelength or wavenumber and drag associated with trapped lee waves from the linear model, as a function of $\left(l_{2} H\right)^{-1}$ and Fr (labeled contours), superposed on the flow regime diagram of Vosper (2004) (adapted from his Fig. 9). (a) $\lambda_{L} l_{2} /(2 \pi)$ and (b) $D_{L} / D_{0}$ for a bell-shaped mountain with $l_{2} a=1.685$; (c) $k_{L} / K$ and (d) $D_{L} / D_{0}$ for a truncated-cosine-shaped mountain with $l_{2} L=12.5$.

$\hat{h}=\frac{h_{0}}{4 \pi K}\left(\frac{2}{k / K}+\frac{1}{1-k / K}-\frac{1}{1+k / K}\right) \sin (\pi k / K)$.

This function attains $e^{-1}$ of its maximum value at $k / K=$ 1.181 , which if $k=1 / a$ means that $a K=0.847$ and thus $a / L=0.135$. This implies that in terms of the mountain half-width $a$ the dimensionless mountain width used by Vosper (2004) is just $l_{2} a=1.685$, which corresponds to a highly nonhydrostatic flow, favorable for the existence of trapped lee waves. This can be confirmed in Figs. 4 and 5, which use relatively similar values of $l_{2} a$. If $a$ and $L$ had been related instead in physical space, taking into account the fact that $a$ is the value of $x$ for which the surface elevation attains half of its maximum, this would yield $a K=\pi / 2$, or $a / L=1 / 4$, so that $l_{2} a=3.125$. While somewhat higher, this still corresponds to clearly nonhydrostatic conditions. However, for the reasons explained above, the equivalence in spectral space has a stronger basis than that in physical space, so $l_{2} a=1.685$ is assumed in the calculations that follow.

Figure 10a shows the normalized trapped-lee-wave wavenumber $\lambda_{L} l_{2} /(2 \pi)$ as a function of $\left(l_{2} H\right)^{-1}$ and $\mathrm{Fr}$ for the range of values employed in Fig. 9 of Vosper (2004), superposed on his flow regime diagram. (Note that the wavelength is a quantity that this author showed to be accurately predicted by linear theory, even in nonlinear flow.) As pointed out by Vosper (2004), trapped lee waves or lee-wave rotors are only observed in his numerical simulations in the region of parameter space below the thick line, which delimits the possibility of occurrence of trapped lee waves according to linear theory. Perhaps more importantly, it can be seen that according to Fig. 10a trapped lee waves or lee-wave rotors (i.e., zones of flow reversal) only could be detected by Vosper (2004) for $\lambda_{L} l_{2} /(2 \pi)>0.3-0.4$. Concerning trapped lee waves, this may be explained by the possibility that very short waves may either be undetectable in the 
numerical simulations owing to resolution limitations, or may have very small amplitude, or be quickly dissipated, either physically or numerically.

In Fig. 10b the normalized trapped-lee-wave drag $D_{L} /$ $D_{0}$ is presented for a bell-shaped mountain with $l_{2} a=$ 1.685 (objectively estimated above), again superposed on the flow regime diagram of Vosper (2004). It can be seen that there is substantial overlap between zones where lee-wave rotors were observed in the numerical simulations of Vosper (2004) and the normalized drag is above 0.01 in the present linear calculations. This suggests a significant correlation between rotors and trapped-lee-wave drag. Physically, this is perhaps not surprising, since trapped-lee-wave drag must be associated with relatively large surface pressure disturbances downstream of the mountain, and Vosper (2004), among others (e.g., Doyle and Durran 2002, 2007), noted that this is a key factor for rotors. The main failure of this correlation is found at the upper and left rim of the nonzero drag region, where neither lee-wave rotors nor even trapped lee waves are present in the regime diagram. This aspect will be further addressed below.

It is worth noting that neither the propagating-wave drag nor the total drag fields (not shown) correlate significantly with any of the flow structures in the diagram [the line defining the linear criterion for wave breaking in the upper layer in Fig. 10 of Vosper (2004), however, qualitatively resembles isolines of the total drag]. Figure 10 of Vosper (2004) presents similar results as his Fig. 9, but for free-slip numerical simulations. Curiously, and for reasons that are not clear, the flow structures shown in that figure (which exclude lee-wave rotors) have a weaker correlation with the linear drag than in Fig. 10b, so the corresponding comparison is not presented here.

It might be argued that it makes more sense to calculate trapped-lee-wave drag for the orography used by Vosper (2004) [see (28)], so that a more direct comparison can be made (the wavelength of the trapped lee waves does not change, since it does not depend on the shape of the orography). In that case, evaluation of the hydrostatic one-layer reference drag $D_{0}$ used to normalize $D_{L}$ becomes more involved, owing to the complicated form of the Fourier transform of the surface elevation in (29). The final result is

$$
D_{0}=1.097 \rho_{0} U^{2} l_{2} h_{0}^{2},
$$

which is slightly larger than (19).

Figures $10 \mathrm{c}$ and $10 \mathrm{~d}$ present the wavenumber of trapped lee waves normalized by $K=2 \pi / L, k_{L} / K$, and $D_{L} / D_{0}$ for the orography described by (28) with $l_{2} L=12.5$, as assumed by Vosper (2004). The Fourier transform (29) has zeros at $k / K=n$, where $n$ is an integer number larger than 1. Consequently, the trapped-lee-wave drag will be zero for $k_{L} / K=n$. The isolines in Fig. 10c therefore signal loci where $D_{L} / D_{0}=0$ according to linear theory. This is confirmed by Fig. 10d. In that figure the isolines of $D_{L} / D_{0}$ extend down to 0.0001 , which appears quite an insignificant value. Nevertheless, with this lower limit, the patch on nonzero trapped-lee-wave drag roughly occupies the region of parameter space where lee-wave rotors exist, and the disagreement pointed out above for the results using a bell-shaped mountain is suppressed. Namely, the nonzero drag region only begins some distance below the line delimiting the existence of trapped lee waves. From Fig. 10c, it can be seen that this is clearly due to the location of the line $k_{L} / K=$ 2 , where necessarily $D_{L} / D_{0}=0$. The drag pattern has other zeros, within the region of parameter space where lee-wave rotors were detected in the numerical simulations, corresponding to $k_{L} / K=3,4,5$.

The overall magnitude of the normalized trapped-leewave drag given by linear theory in Fig. 10d is very low (at most about 0.02). This is due to the Fourier transform (29) having a much lower absolute value than the Fourier transform (16) in the region surrounding its first zeros (which gives a dominant contribution to the drag for the assumed value of $l_{2} L$ ). As pointed out by Vosper (2004), in nonlinear conditions this picture changes, because the trapped lee waves are not forced only directly by the orography, so the zeros in the forcing spectrum are suppressed, and the magnitude of the spectrum in the forcing region increases. Thus, the form of the orography employed, rather than intrinsic shortcomings of linear theory, may account for a sizeable fraction of the underestimation of the trapped-lee-wave amplitude by linear theory in Vosper (2004). The Fourier transform of the bell-shaped mountain adopted here [see (16)] does not have any zeros, so in that sense it seems more representative of real flows, perhaps allowing the drag to be predicted more accurately up to higher values of $l_{2} h_{0}$. Nevertheless, both foregoing results are useful for stressing the potential link between trapped-lee-wave drag and rotors - an aspect that certainly deserves more detailed study.

\section{Concluding remarks}

The drag force associated with trapped lee waves generated at a temperature inversion in flow over a $2 \mathrm{D}$ mountain ridge was calculated analytically, to the authors' knowledge for the first time. The atmosphere was assumed to be stably stratified above the inversion, but neutral below, mimicking the effect of a well-mixed 
boundary layer. Apart from trapped-lee-wave drag, drag associated with waves propagating in the upper layer is also produced. Both drag components, when normalized by the hydrostatic drag for a one-layer atmosphere equal to the upper layer, take values that may substantially exceed 1 , and depend on three parameters: the Froude number of the flow (Fr), the dimensionless inversion height $\left(l_{2} H\right)$, and the dimensionless width of the ridge $\left(l_{2} a\right)$.

Both components of the drag are found to decrease as $l_{2} \mathrm{H}$ increases, because the interaction of the orography with the inversion, in the case of trapped lee waves, and with the stable layer above, in the case of vertically propagating waves, weakens when these atmospheric structures are lifted away from the surface. Trapped lee waves only occur for $\mathrm{Fr}<1$, but both components of the drag attain their maximum values near $\mathrm{Fr}=1$. In the case of trapped lee waves, this happens when the incoming flow velocity matches the propagation speed of interfacial gravity waves (which reduce to "shallow water" waves in the limit $l_{2} H \rightarrow 0$ ), leading to resonance. For waves propagating in the upper stable layer, the drag maximum at $\mathrm{Fr}=1$ can be attributed to the amplification of the wave-associated buoyancy perturbation in the upper layer that takes place when the potential temperature is discontinuous at $z=H$. This effect loses relevance for lower Fr because the temperature discontinuity then becomes too large for effective transmission of the vertical velocity perturbation to the upper layer (in that case the inversion acts more like a rigid lid).

The propagating-wave drag decreases as the flow becomes more nonhydrostatic (i.e., $l_{2} a$ decreases), as happens in a single-layer atmosphere, but for physically reasonable values of $l_{2} H$ the trapped-lee-wave drag, on the contrary, attains its maximum values for $l_{2} a=O(1)$. Contributions to trapped-lee-wave drag come from wavenumbers higher than $l_{2}$ (i.e., relatively small horizontal scales), and if the trapped lee waves are short enough their associated pressure perturbations alternate in sign many times over the downwind slope of the ridge, so their contribution is likely to cancel out. However, this cancellation does not occur when $l_{2} a=O(1)$, because then the wavelength of the pressure fluctuations associated with the trapped lee waves (which should be similar to the wavelength of the waves themselves) roughly matches the width of the ridge. A related effect had previously been gleaned by Grisogono et al. (1993) and Vosper (1996), and was elaborated more recently by Grubišić and Stiperski (2009) and Stiperski and Grubišić (2011), when they stressed the importance of the ratio of the spacing between two consecutive mountains and the wavelength of trapped lee waves as a key flow parameter. Clearly, when this ratio is $O(1)$, the spectral amplitude of the orography and of the trapped lee waves have a coinciding maximum, and a highamplitude wave response is expected.

Unlike what the previous reasoning might suggest, the ratio between the wavelength of trapped lee waves and the mountain half-width $\lambda_{L} / a$ is not an explicit parameter of the flow described by the present model. However, since the highest trapped-lee-wave drag values occur when $\lambda_{L} \approx l_{2}^{-1}$, this dependency is in fact encapsulated in $l_{2} a$. It is worth noting that in the twolayer model of Teixeira et al. (2013) the values of $l_{1} a$ used to illustrate the trapped-lee-wave drag behavior were 10,5 , and 2 (where $l_{1}$ is the Scorer parameter in the lower layer), corresponding (for $l_{2} / l_{1}=0.2$ assumed by them) to $l_{2} a=2,1$, and 0.4 , respectively. The fact that these values are within the range employed in the present study further corroborates the assertion that trapped-lee-wave drag is maximized for $l_{2} a=O(1)$.

Comparisons of results from the present model with the regime diagram of Vosper (2004), showing the flow structures observed in numerical simulations of trapped lee waves, reveal that rotors mostly occur for waves with the largest wavelengths, which produce most of the trapped-lee-wave drag. This is perhaps not surprising since rotors, while being intrinsically nonlinear structures associated with flow stagnation and reversal, are triggered by wave amplification, which, to a large extent, can be understood in the framework of linear theory. Therefore, it makes sense that the drag, which is an integral measure of the wave intensity, correlates well with these structures.

The analytical expression obtained in the present study for trapped-lee-wave drag provides a benchmark against which the drag from more-refined models may be gauged. This new result is potentially important for guiding the formulation of improvements to existing drag parameterization schemes. While the effect on the atmosphere of the drag associated with vertically propagating waves may act at high levels, as in a single-layer atmosphere, the reaction force to trappedlee-wave drag will necessarily be felt at the inversion height, probably giving a sizeable contribution to the low-level flow deceleration in situations of practical interest.

A natural next step in the line of research initiated here would be using nonlinear numerical simulations, with and without friction, to assess the limitations of the analytical drag expression derived here from linear theory. There is also ample scope for using both methodologies to further investigate the onset of flow stagnation and reversal associated with trapped lee waves-a 
problem directly relevant to rotors and their applications in aviation meteorology.

Acknowledgments. The authors acknowledge the financial support of the Portuguese Science Foundation (FCT) under Grants PEst-OE/CTE/LA0019/2011 and PTDC/CTE-ATM/122501/2010.

\section{APPENDIX}

\section{Details of the Numerical Simulations}

The numerical simulations presented in section 3 used the mesoscale-to-microscale model FLEX (Argaín et al. 2009). This model is $2 \mathrm{D}$, nonlinear, and nonhydrostatic, and was run here in inviscid and nonrotating mode. For all simulations, the wind speed was $U=10 \mathrm{~m} \mathrm{~s}^{-1}$; the Brunt-Väisälä frequency in the upper layer was $N_{2}=0.01 \mathrm{~s}^{-1}$, giving $l_{2}=10^{-3} \mathrm{~m}^{-1}$; and the ridge height was $h_{0}=10 \mathrm{~m}$. The thickness of the neutral layer was $H=500 \mathrm{~m}$ most of the time, corresponding to $l_{2} H=0.5$. The mountain widths considered were $a=200,500,1000,2000$, and $5000 \mathrm{~m}$. This gives $l_{2} h_{0}=0.01$ and $h_{0} / a=0.05,0.02,0.01,0.005$, and 0.002 , respectively (i.e., highly linear conditions), and $l_{2} a=0.20 .5,1,2$, and 5, respectively, as required. The temperature jump at the inversion varied between $\Delta \theta=0.75$ and $65.31 \mathrm{~K}$, to obtain Froude numbers Fr between 0.3 and 2.8 (in order to adequately resolve the drag maxima). The largest values of $\Delta \theta$ do not strictly satisfy the assumption $\Delta \theta / \theta_{0} \ll 1$, used in the derivation of the boundary condition (8), but that is not a very serious limitation, because for the corresponding values of Fr the drag is very small.

The domain extent in the horizontal direction was $50 a$ (10a upstream of the mountain and $40 a$ downstream of it) in all cases, and about $35 \mathrm{~km}$ in the vertical, which corresponds to approximately 5.5 hydrostatic wavelengths in the upper layer. The grid spacing in the vertical was $\Delta z=20 \mathrm{~m}$ in all cases, and the horizontal grid spacing was $\Delta x=100 \mathrm{~m}$, except in the case with $l_{2} a=2$, for which $\Delta x=40 \mathrm{~m}$. This was done to ensure that both the mountain width and the wavelength of the trapped lee waves were conveniently resolved in the horizontal and the upward-propagating waves were resolved in the vertical. Sponges spanning 15 grid points at the upwind boundary and 30 grid points at the downwind boundary, and spanning two hydrostatic vertical wavelengths at the upper boundary, were employed.

The time step used in the integration was $\Delta t=0.5,0.5$, $1.0,1.0$, and $2.0 \mathrm{~s}$, respectively, for $l_{2} a=0.2,0.5,1,2$, and 5. The integration time was $500 a / U$ in all cases, ranging between about $2.8 \mathrm{~h}$ for $l_{2} a=0.2$ and $69.4 \mathrm{~h}$ for $l_{2} a=5$. After this time, the drag stabilized to a nearly constant value, and the average of the last few time steps was taken as representative.

\section{REFERENCES}

Argaín, J. L., P. M. A. Miranda, and M. A. C. Teixeira, 2009: Estimation of the friction velocity in stably stratified boundarylayer flows over hills. Bound.-Layer Meteor., 130, 15-28.

Baines, P. G., 1995: Topographic Effects in Stratified Flows. Cambridge University Press, 498 pp.

Bretherton, F. P., 1969: Momentum transport by gravity waves. Quart. J. Roy. Meteor. Soc., 95, 213-243.

Broutman, D., J. W. Rottman, and S. D. Eckermann, 2002: Maslov's method for stationary hydrostatic mountain waves. Quart. J. Roy. Meteor. Soc., 128, 1159-1171.

,-- , and - , 2003: A simplified Fourier method for nonhydrostatic mountain waves. J. Atmos. Sci., 60, 2686-2696.

Chimonas, G., and C. J. Nappo, 1989: Wave drag in the planetary boundary layer over complex terrain. Bound.-Layer Meteor., 47, 217-232.

Darby, L. S., and G. S. Poulos, 2006: The evolution of lee-waverotor activity in the lee of Pike's Peak under the influence of a cold frontal passage: Implications for aircraft safety. Mon. Wea. Rev., 134, 2857-2876.

Doyle, J. D., and D. R. Durran, 2002: The dynamics of mountainwave-induced rotors. J. Atmos. Sci., 59, 186-201.

- , and -2007 : Rotor and subrotor dynamics in the lee of three-dimensional terrain. J. Atmos. Sci., 64, 4202-4221.

Grisogono, B., 1994: Dissipation of wave drag in the atmospheric boundary layer. J. Atmos. Sci., 51, 1237-1243.

_ - S. C. Pryor, and R. E. Keislar, 1993: Mountain wave drag over double bell-shaped orography. Quart. J. Roy. Meteor. Soc., 119, 199-206.

Grubišić, V., and I. Stiperski, 2009: Lee-wave resonances over double bell-shaped obstacles. J. Atmos. Sci., 66, 1205-1228.

Lin, Y.-L., 2007: Mesoscale Dynamics. Cambridge University Press, 674 pp.

Mobbs, S. D., and Coauthors, 2005: Observations of downslope winds and rotors in the Falkland Islands. Quart. J. Roy. Meteor. Soc., 131, 329-351.

Reinecke, P. A., and D. R. Durran, 2008: Estimating topographic blocking using a Froude number when the static stability is nonuniform. J. Atmos. Sci., 65, 1035-1048.

Scorer, R. S., 1949: Theory of waves in the lee of mountains. Quart. J. Roy. Meteor. Soc., 75, 41-56.

— 1953: Theory of airflow over mountains: II-The flow over a ridge. Quart. J. Roy. Meteor. Soc., 79, 70-83.

_ 1954: Theory of airflow over mountains: III-Airstream characteristics. Quart. J. Roy. Meteor. Soc., 80, 417-428.

Sheridan, P. F., and S. B. Vosper, 2006a: A flow regime diagram for forecasting lee waves, rotors and downslope winds. Meteor. Appl., 13, 179-195.

$\longrightarrow$, and $-2006 \mathrm{~b}$ : Numerical simulations of rotors, hydraulic jumps and eddy shedding in the Falkland Islands. Atmos. Sci. Lett., 6, 211-218.

Smith, R. B., 1976: The generation of lee waves by the Blue Ridge. J. Atmos. Sci., 33, 507-519.

, 1980: Linear theory of stratified hydrostatic flow past an isolated obstacle. Tellus, 32, 348-364.

Stiperski, I., and V. Grubišić, 2011: Trapped lee wave interference in presence of surface friction. J. Atmos. Sci., 68, 918-936. 
Teixeira, M. A. C., and P. M. A. Miranda, 2005: Linear criteria for gravity-wave breaking in resonant stratified flow over a ridge. Quart. J. Roy. Meteor. Soc., 131, 1815-1820.

_ , and _ 2006: A linear model of gravity wave drag for hydrostatic sheared flow over elliptical mountains. Quart. J. Roy. Meteor. Soc., 132, 2439-2458.

— , and — 2009: On the momentum fluxes associated with mountain waves in directionally sheared flows. J. Atmos. Sci., 66, 3419-3433.

- - — and J. L. Argaín, 2008: Mountain waves in two-layer sheared flows: Critical level effects, wave reflection, and drag enhancement. J. Atmos. Sci., 65, 1912-1926.
— J. L. Argaín, and P. M. A. Miranda, 2012: The importance of friction in mountain wave drag amplification by scorer parameter resonance. Quart. J. Roy. Meteor. Soc., 138, 1325-1337.

,-- , and,- 2013 : Drag produced by trapped lee waves and propagating mountain waves in a two-layer atmosphere. Quart. J. Roy. Meteor. Soc., 139, 964-981.

Vosper, S., 1996: Gravity-wave drag on two mountains. Quart. J. Roy. Meteor. Soc., 122, 993-999.

_ 2004: Inversion effects on mountain lee waves. Quart. J. Roy. Meteor. Soc., 130, 1723-1748.

Wurtele, M. G., R. D. Sharman, and A. Datta, 1996: Atmospheric lee waves. Annu. Rev. Fluid Mech., 28, 429-476. 